\title{
Clinical applications of the indirect immunofluorescence assay for detection of anticell membrane-associated DNA antibodies in juvenile systemic lupus erythematosus
}

Jin-li Ru', Yue Zhao', Xiao-xiang Xie' ${ }^{1}$, Guo-zhu Che' ${ }^{1}$ Chuan-fang Cheng ${ }^{1}$, Hua-ming Zhao' ${ }^{1}$ Zhi-yong Jin ${ }^{1}$, Hui-ping Sun ${ }^{1}$ and Xiao-feng $\mathrm{Li}^{2}$

BACKGROUND: Juvenile-onset systemic lupus erythematosus (JSLE) has a higher mortality risk compared to adultonset SLE. We compared the diagnostic value of anti-cmDNA antibodies with that of antinucleosome antibodies (AnuA), anti-Sm antibodies, and anti-dsDNA antibodies and human B lymphocyte Raji cells with that of human promyelocytic leukemia HL60 cells as substrates in an indirect immunofluorescence assay to detect anti-cmDNA antibodies in JSLE patients. METHODS: We recruited 92 JSLE patients and 71 patients with other juvenile-onset rheumatic diseases. Anti-cmDNA antibodies and antinuclear antibodies (ANA) were detected in patient sera using indirect immunofluorescence assays. Anti-dsDNA antibodies were detected by combining ELISA and indirect immunofluorescence. Anti-Sm antibodies were detected by double immunodiffusion assay and immunoblotting, while AnuA were detected by ELISA.

RESULTS: The JSLE group had a significantly higher percentage of patients positive for anti-cmDNA compared to patients with other rheumatoid diseases. Using one antibody for diagnosis, anti-cm DNA antibodies had the highest accuracy at 84.0\%; using two antibodies, the combination of anti-cm DNA and anti-dsDNA antibodies had 90.8\% accuracy. Raji cells used as substrate demonstrated a stronger intensity of fluorescent patterns compared to HL60 cells.

CONCLUSION: The high sensitivity, specificity, and accuracy of detection of anti-cmDNA antibodies make it a valuable diagnostic tool for JSLE.

S ystemic lupus erythematosus (SLE) is an autoimmune disease characterized by the presence of autoimmune antibodies against a number of nuclear and nonnuclear antigens, resulting in immune complex-mediated inflammation and systemic, multiple organ failure (1). Almost $15-20 \%$ of all SLE patients are below the age of 18 (ref. 2). Juvenile SLE (JSLE) is characterized by an exaggerated immune response with highly activated autoreactive B lymphocytes, dysregulated immune regulatory pathways, and frequent involvement of the renal, hematological and central nervous systems (3-5). Children with JSLE also have a higher disease severity and a significantly higher mortality risk compared to patients with adult onset disease $(6,7)$. Timely diagnosis and treatment have been shown to significantly improve the prognosis and the clinical management of JSLE (8).

Disease activity in SLE patients is currently evaluated using the SLE activity index (SLEDAI), the systemic lupus activity measure, and the British Isles Lupus Assessment Group (9). Recently, the anti-Smith (Sm) antibodies, anticell membrane DNA (cmDNA) antibodies, antidouble strand DNA (dsDNA) antibodies, antinuclear antigen (ANA) antibodies, antinucleosome (AnuA) antibodies, anti-C1q antibodies, and antiphospholipid antibodies have been proposed as useful biomarkers of JSLE (10-16). Anti-dsDNA antibodies, anti-Sm antibodies and antiphospholipid antibodies are included in the diagnostic criteria outlined by the American College of Rheumatology (10). JSLE patients were shown to be more frequently positive for anti-dsDNA antibodies, antiribosomal $\mathrm{P}$ antibodies, antiSm antibodies, and antihistone antibodies compared to adult SLE patients $(17,18)$.

Although most antigenic DNA is localized in the nucleus, a $17 \mathrm{kB}$ antigenic cell membrane-associated DNA (cmDNA) has been found on the outer leaflet of the plasma membrane of B lymphocytes and monocytes (19-22). cmDNA is a target for autoantibodies in SLE patients (13). We recently established an indirect immunofluorescence assay to measure anti-cmDNA antibodies using human promyelocytic leukemia cells (HL60) as a substrate for the assay (16). Using this assay, we showed high levels of anti-cmDNA antibodies in JSLE patients, including those who were negative for anti-dsDNA and anti-Sm antibodies (16), suggesting that anti-cmDNA antibodies could be an efficient diagnostic biomarker for JSLE.

In this study, we validated the diagnostic value of anticmDNA antibodies in JSLE patients using HL60 as well as human B lymphocyte Raji cells as substrates in our indirect immunofluorescence assay. We also investigated the correlation between anti-cmDNA antibodies and clinical features, laboratory parameters and SLEDAI scores in JSLE patients. 


\section{RESULTS}

\section{Prevalence of Anti-cmDNA in JSLE Patients}

Serum from a total of 163 patients (including 92 JSLE patients and 71 patients with other juvenile rheumatoid diseases) were incubated with Raji cells. The JSLE group had a significantly higher percentage of patients positive for anticmDNA $(69 / 92,75 \%)$ compared to the juvenile spondyloarthritis group $(1 / 32,3.1 \%)$, the juvenile idiopathic arthritis group $(1 / 18,5.6 \%)$, the juvenile dermatomyositis and juvenile polymyositis groups $(0 / 5,0 \%)$, the juvenile systemic sclerosis group $(0 / 4,0 \%)$, the pediatric vasculitis group $(0 / 2,0 \%)$ and the juvenile connective tissue disease group (1/10, 10\%), respectively $(P<0.001)$. Similarly, of the 163 patients whose sera were incubated with HL-60 cells, the JSLE group had a significantly higher percentage of patients positive for anticmDNA $(73 / 92,79.4 \%)$ compared to the juvenile spondyloarthritis group $(2 / 32,6.3 \%)$, the juvenile idiopathic arthritis group $(3 / 18,16.7 \%)$, the juvenile dermatomyositis/juvenile polymyositis group $(0 / 5,0 \%)$, the juvenile systemic sclerosis group $(0 / 4,0 \%)$, the pediatric vasculitis group $(0 / 2,0 \%)$, and the juvenile connective tissue disease group (3/10, 30\%), respectively $(P<0.001)$.

\section{Frequency of Anti-cmDNA in JSLE Patients Negative for Anti-} dsDNA, Anti-Sm, AnuA, and ANA

The frequency of anti-cmDNA positivity in JSLE patients lacking anti-dsDNA antibodies, anti-Sm antibodies, AnuA and

Table 1. Frequency of anti-cmDNA antibodies in patients who were negative for anti-dsDNA, anti-Sm, AnuA, and ANA antibodies

\begin{tabular}{llcr}
\hline \multirow{2}{*}{ Antibodies } & \multicolumn{3}{c}{$\%$ Anti-cmDNA positivity } \\
\cline { 2 - 4 } Anti-dsDNA (-) & $25.9(28 / 108)$ & $67.6(25 / 37)$ & $4.2(3 / 71)$ \\
Anti-Sm (-) & $29.5(36 / 122)$ & $64.7(33 / 51)$ & $4.2(3 / 71)$ \\
AnuA (-) & $22.0(22 / 100)$ & $67.7(21 / 31)$ & $1.5(1 / 69)$ \\
ANA (-) & $17.7(11 / 62)$ & $73.3(11 / 15)$ & $0(0 / 47)$ \\
\hline
\end{tabular}

$(-)$, Antibody negative. Data are presented as percentage of anti-cmDNA positivity in the different antibody-negative groups.
ANA was 67.6, 64.7, 67.7, and 73.3\%, respectively (Table 1). There were 11 patients who were negative for both ANA and anti-dsDNA antibodies, and diagnosed with JSLE based only on clinical criteria.

Sensitivity and Specificity of Diagnosis With Different Antibodies The sensitivity and specificity of JSLE diagnosis using anticmDNA antibodies were 75.0 and $95.8 \%$, respectively. The positive and negative predictive values of anti-cmDNA antibodies for JSLE were 95.8 and $74.7 \%$, respectively. The accuracy of anti-cmDNA antibodies for all 163 patients was $84.0 \%$. The accuracy of diagnosis of all 163 patients using anti-cmDNA was significantly higher compared to that using anti-Sm antibodies ( 84.0 vs. $68.7 \%, P=0.002)$. But, there was no significant difference between anti-cmDNA and the other antibodies in accuracy of diagnosis of all 163 patients. (Table 2)

The Correlation of Anti-cmDNA With Clinical Manifestations and Laboratory Parameters in JSLE Patients

Patients who were positive for anti-cmDNA antibodies showed no significant differences in clinical features such as skin rash, alopecia, fever, or oral ulcers compared to patients who were negative for anti-cmDNA antibodies.

The anti-cmDNA negative and positive groups also did not differ significantly in laboratory parameters and SLEDAI scores (Table 3 ).

\section{Comparison of Raji and HL60 Cells as Substrates for Indirect} Immunofluorescence Assays to Detect Anti-cmDNA Antibodies

We used Raji and HL60 cells as substrates in indirect immunofluorescence assays to detect anti-cmDNA antibodies. We showed that patients who were positive for anti-cmDNA antibodies exhibited continuous fluorescence patterns that were distributed along the cell membrane. Although Raji and HL60 cells are morphologically similar, Raji cells demonstrated a stronger intensity of fluorescent patterns, making it the substrate cell line of choice for indirect immunofluorescence (Figure 1).

Table 2. Antibody profiles in all 163 study patients (92 patients with JSLE and 71 patients with other rheumatoid diseases)

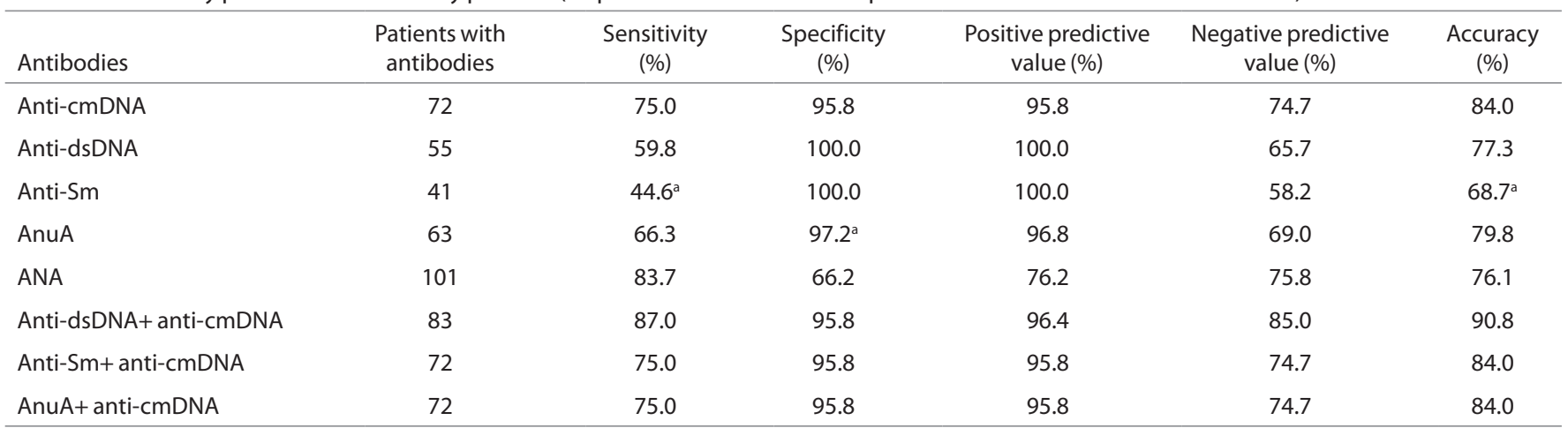

The $\chi^{2}$ test was compared the diagnostic accuracy of the different antibodies. The McNemar's test was provided to measure the consistency of sensitivity and specificity in difference antibodies.

asignificantly different from anti-cmDNA antibody $(P<0.05)$. 
Table 3. Correlation of anti-cmDNA antibodies and clinical features in JSLE patients

\begin{tabular}{|c|c|c|c|}
\hline & $\begin{array}{c}\text { Anti-cmDNA } \\
\text { negative }(n=23)\end{array}$ & $\begin{array}{c}\text { Anti-cmDNA } \\
\text { positive }(n=69)\end{array}$ & $P$ value \\
\hline \multicolumn{4}{|l|}{ Clinical features } \\
\hline Skin rasha & $17(73.9 \%)$ & $47(68.1 \%)$ & 0.794 \\
\hline Alopecia $^{a}$ & $5(21.7 \%)$ & $25(36.2 \%)$ & 0.304 \\
\hline Fever $^{\mathrm{a}}$ & $9(39.1 \%)$ & $38(55.1 \%)$ & 0.278 \\
\hline Oral ulcer ${ }^{\mathrm{a}}$ & $10(43.5 \%)$ & $21(30.4 \%)$ & 0.373 \\
\hline $\begin{array}{l}\text { Neurologic } \\
\text { manifestations }{ }^{\mathrm{a}}\end{array}$ & $5(21.7 \%)$ & $14(20.3 \%)$ & 0.882 \\
\hline $\begin{array}{l}\text { Cutaneous } \\
\text { vasculitis }^{\mathrm{a}}\end{array}$ & $8(34.8 \%)$ & $12(17.4 \%)$ & 0.080 \\
\hline Arthritis ${ }^{a}$ & $10(43.5 \%)$ & $23(33.3 \%)$ & 0.380 \\
\hline \multicolumn{4}{|l|}{ Laboratory parameters } \\
\hline Hematuriaa $^{a}$ & $6(26.1 \%)$ & $30(43.5 \%)$ & 0.217 \\
\hline Proteinuria $^{a}$ & $13(56.5 \%)$ & $39(56.5 \%)$ & 1.000 \\
\hline $\mathrm{CRP}^{\mathrm{b}}$ & $3.4(1.0,9.4)$ & $4.5(2.0,9.4)$ & 0.438 \\
\hline Leukopenia $^{\mathrm{b}}$ & $3.8(3.0,4.9)$ & $4.9(2.7,7.3)$ & 0.433 \\
\hline Thrombocytopeniab & $139.0(69.5,227.0)$ & $147.1(101.4,231.1)$ & 0.698 \\
\hline$C 3^{c}$ & $0.63 \pm 0.36$ & $0.58 \pm 0.35$ & 0.572 \\
\hline$C 4^{c}$ & $0.15 \pm 0.11$ & $0.14 \pm 0.08$ & 0.862 \\
\hline $\mathrm{ESR}^{\mathrm{b}}$ & $36.0(20.0,84.0)$ & $54.0(30.0,82.0)$ & 0.749 \\
\hline SLEDAI $^{\mathrm{b}}$ & $16.0(10.0,22.0)$ & $15.0(11.0,23.0)$ & 0.907 \\
\hline
\end{tabular}

CRP, C-reactive protein; ESR, erythrocyte sedimentation rate.

${ }^{2}$ Categorical variables were expressed by counts and percentages, and compared between different groups by the $\chi^{2}$ test. ${ }^{b}$ Continuous variables were presented as medians and interquartile ranges (the range between the 25 th and 75 th percentile) due to nonnormal distribution, and analyzed by the Mann-Whitney U-test. 'Continuous variables were presented as mean and standard deviation due to normal distribution, and analyzed by the Student's $t$-test.

\section{DISCUSSION}

In this study, we compared the diagnostic value of using anticmDNA antibodies with that of AnuA, anti-Sm and antidsDNA antibodies for JSLE. We also compared Raji and HL60 cell lines as substrates for indirect immunofluorescence assays to detect anti-cmDNA antibodies in JSLE patients. We showed that anti-cmDNA antibodies had a significantly higher diagnostic sensitivity compared to anti-Sm antibodies, and a significantly higher diagnostic specificity compared to ANA. The Raji cell line, which exhibited a higher intensity of fluorescence compared to HL60 cells, was the substrate of choice for indirect immunofluorescence assays.

There has been a recent focus on identifying diagnostic markers for JSLE, including AnuA, anti-cmDNA antibodies, anti-dsDNA antibodies, anti-Sm antibodies, antinuclear antibodies, and anti-C1q antibodies $(12,13,23,24)$. Although antinuclear antibodies are found in 90\% of JSLE patients (25), almost $33 \%$ of healthy children also showed positive ANA titres (26). Serum levels of AnuA were diagnostic and correlated with disease severity in JSLE patients $(14,23)$. Dysfunction of the 30 $\mathrm{KDa}$ cell membrane receptor of cmDNA was previously shown to prevent cmDNA clearance in SLE patients, with subsequent production of anti-cmDNA antibodies $(27,28)$. Although JSLE

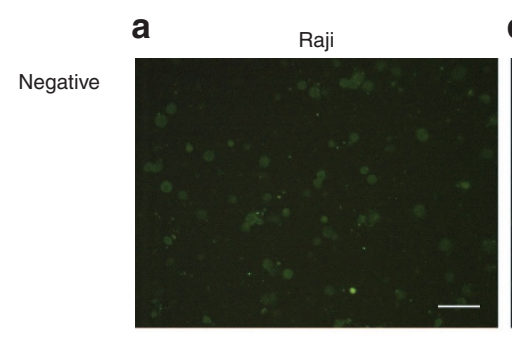

d $\quad$ HL6O
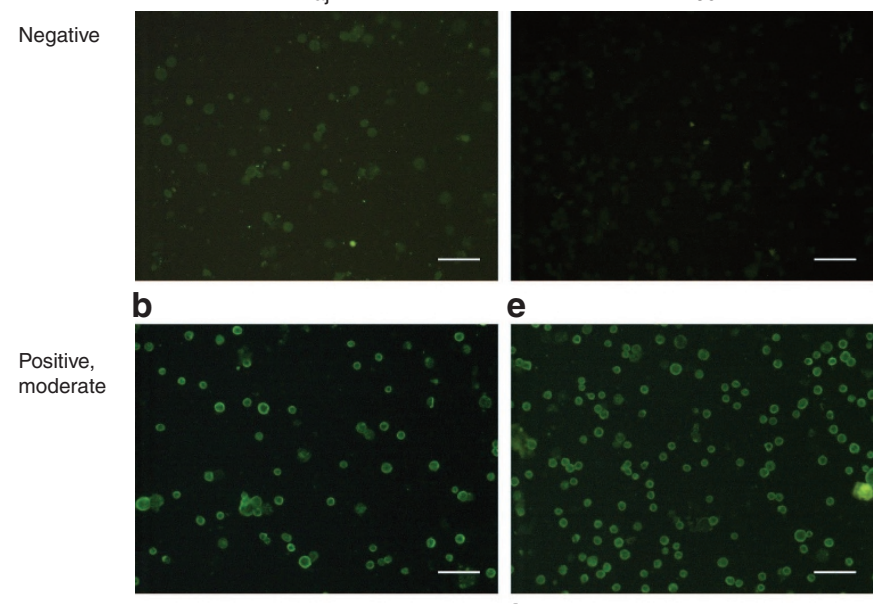

e
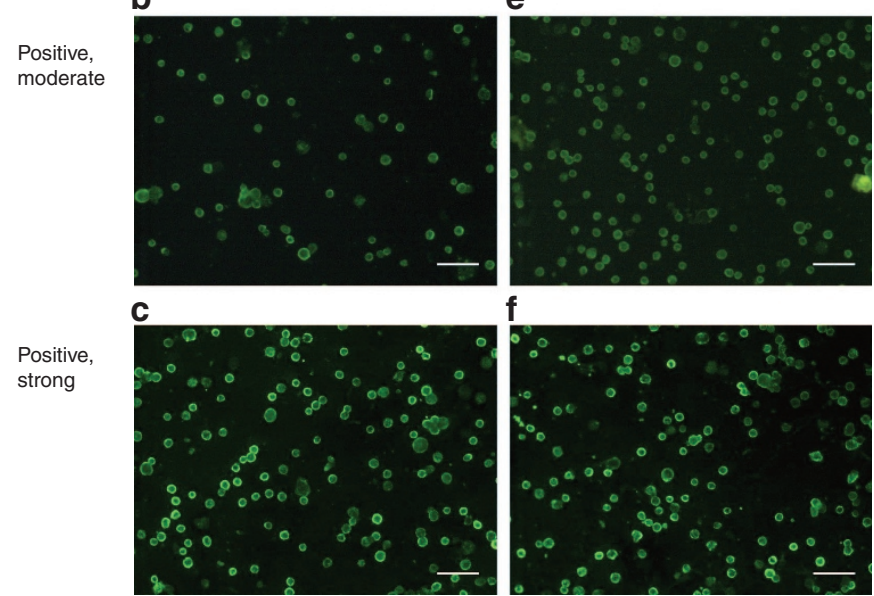

Figure 1. Immunofluorescence images in different substrate cells. Detection of anti-cmDNA antibodies using indirect immunofluorescence with Raji or HL60 cells (20x). (a-c) Raji cells that stained negative, moderately positive, and strongly positive, respectively. (d-f) HL60 cells that stained negative, moderately positive, and strongly positive, respectively. The scale bars represent $80 \mu \mathrm{m}$.

patients have a higher positivity rate for anti-cmDNA antibodies compared to patients with other rheumatoid diseases or healthy controls $(13,27-32)$, the role of anti-cmDNA antibodies in JSLE remains unclear. To the best of our knowledge, we are the first to report that anti-cmDNA antibodies can be used for diagnosis of JSLE with a sensitivity of $77.3 \%$ and specificity of $80.0 \%$.

A number of B lymphocyte cell lines, including Raji, Priess, Daudi, BJAB, and 3D5, T lymphocyte cell lines including Jurkat and chronic myeloid leukemia K562, and promyelocytic leukemic HL60 cells have been used as substrates in indirect immunofluorescence assays to detect anti-cmDNA antibodies (29-31). However, ours is the first report that directly compared the diagnostic values of Raji and HL60 cells as substrates for the indirect immunofluorescence assay. We showed that although there was no significant difference in the sensitivity and specificity of detection of anti-cmDNA antibodies between the two cell lines, Raji cells exhibited more intense fluorescent patterns and more stable test results compared to HL60 cells. Ease of thawing, rapid growth, and lower cost were other advantages of using Raji cells.

Our sensitivity and specificity data using Raji cells as well as HL60 cells were consistent with previous results $(20,31)$. However, the sensitivity of detection of anti-cmDNA antibodies in our study was higher compared to a study that used Wil2 cells as a substrate to detect anti-cmDNA antibodies in SLE patients (13). It will be interesting to investigate if the higher sensitivity 
seen in our study could reflect (i) differences in pathogenic mechanisms and disease progression, (ii) different effects of anti-cmDNA antibodies in adult SLE and JSLE patients, (iii) substrate cell line, or (iv) differences in race of the study patients.

Using Raji cells as substrate, the sensitivity of detection of anticmDNA antibodies was significantly higher than that of anti-Sm antibodies but was not significantly different from that of antidsDNA antibodies. There was no significant difference in the specificity of detection of anti-cmDNA antibodies, anti-dsDNA antibodies, anti-Sm antibodies and AnuA. Although the sensitivity of detection of ANA was higher than that of anti-cmDNA antibodies, the specificity of detection of ANA was significantly lower than that of anti-cmDNA antibodies. The accuracy of combined detection of both anti-dsDNA and anti-cmDNA antibodies was as high as $90.8 \%$. Using HL60 cells, we previously showed that the positivity rates of anti-cmDNA antibodies in JSLE patients who were negative for anti-dsDNA antibodies and anti-Sm antibodies were 68 and 79\%, respectively (16). Our present study which has a larger study population, included all the patients from our previous study. Our present data combined with our previous results, suggest that detection of anti-cmDNA antibodies has a high diagnostic value in JSLE diagnosis.

In summary, we showed that detection of anti-cmDNA antibodies using indirect immunofluorescence is a highly sensitive and specific diagnostic test for JSLE. It is important to note that due to the complex pathogenesis of JSLE, the sensitivity of JSLE diagnosis can be enhanced by using a combination of biomarkers such as anti-cmDNA, anti-dsDNA, anti-Sm, and antinucleosome antibodies.

\section{METHODS}

\section{Study Subjects}

This prospective, multicenter study recruited 92 juvenile outpatients as well as inpatients with SLE from the Chinese People's Liberation Army 264th Hospital and Shanxi Medical University Second Hospital Division of Rheumatology between January 2007 and December 2010. The study population comprised 13 males and 79 females, aged $9-16$ with an average age of $14.3 \pm 2.5$, and a mean disease duration of $14.9 \pm 9.4$ mo. The study also recruited 71 juvenile patients (comprising 27 males and 45 females) with other rheumatic diseases, aged 7-16 with an average age of $12.6 \pm 19.6$, and an average disease duration of $13.3 \pm 20.9$ mo. Of the 71 patients with other rheumatoid diseases, there were 31 cases of juvenile spondyloarthritis, 18 cases of juvenile idiopathic arthritis, 5 cases of juvenile dermatomyositis and juvenile polymyositis, 4 cases of juvenile systemic sclerosis, 10 cases of pediatric vasculitis, 2 cases of juvenile connective tissue disease, and 1 case of still disease. Diagnosis of JSLE was based on the American College of Rheumatology Revised Criteria for Classification of Systemic Lupus Erythematosus published in 1997, which includes the presence of 11 symptoms, clinical signs, and laboratory testing. In this study, diagnosis of SLE was dependent on the presence of four or more of the 11 criteria. All other diseases were diagnosed in accordance with the corresponding international diagnostic classification criteria. Informed consents were obtained from the guardians of all the study subjects, and the study was approved by the Institutional Review Boards of the Chinese People's Liberation Army 264th Hospital and Shanxi Medical University Second Hospital.

\section{Detection of Anti-cmDNA Antibodies by Indirect Immunofluorescence}

cmDNA specificity of the antibodies was confirmed by pattern extinction using DNAse, RNase and protease pretreatment. Briefly, the slides were fixed and then incubated for $16 \mathrm{~h}$ at room temperature with RNase-free DNase RQ1 $(25 \mu \mathrm{g}$ in $25 \mu \mathrm{l}$ phosphate-buffered solution (PBS); Takara Dalian, Dalian, Liaoning Province, China), and then incubated for $16 \mathrm{~h}$ at $37^{\circ} \mathrm{C}$ with RNase $(2.5 \mu \mathrm{g}+25 \mu \mathrm{l}$ PBS, Sigma-Aldrich, Beijing, China). The slides were then incubated for $30 \mathrm{~min}$ at $37^{\circ} \mathrm{C}$ with protease $(3.125 \mu \mathrm{g}+25 \mu \mathrm{l}$ PBS, Sigma-Aldrich).

Human B lymphocyte Raji cells (Boster, Wuhan, China) and human promyelocytic leukemia HL60 cells (Institute of Hematology, Chinese Academy of Medical Sciences, Tianjin City, China) were cultured in RPMI 1640 medium (Gibco, Invitrogen, Carlsbad, CA) supplemented with $10 \%$ fetal calf serum. Cells were maintained at $37^{\circ} \mathrm{C}$ in the presence of $5 \% \mathrm{CO}_{2}$. Cells in the exponential growth phase (density of $(1-1.2) \times 10^{6} / \mathrm{ml}$ ) were harvested by centrifugation, washed twice with PBS (10 mmol/l, $\mathrm{pH} 7.2)$, resuspended to achieve a density of $0.5 \times 10^{6} / \mathrm{ml}$, and $15 \mu \mathrm{l} \mathrm{vol-}$ umes were plated on separate slides. The slides were dried at room temperature, fixed in methanol for $3 \mathrm{~min}$, then washed once with PBS (10 mmol/l, pH 7.2) and allowed to air dry. Subsequently, the slides were incubated with diluted fetal calf serum (fetal calf serum/ PBS: 1:5) for $5 \mathrm{~min}$, and then washed once with buffer solution (PBS-0.2\% Tween 20) and PBS, respectively, to prevent nonspecific binding of serum or fluorescence-bound IgG to the cells. The IF-LUPUS diagnostic kit (Biotech Tools Company, Belgium) was used according to the manufacturer's instructions. Briefly, the sera were diluted 1:40 with $0.2 \%$ PBS-Tween 20 , and incubated with Raji and HL60 cells for $30 \mathrm{~min}$ at room temperature. The slides were washed once with $0.2 \%$ PBS-Tween 20 and twice with PBS, and then incubated for $30 \mathrm{~min}$ at room temperature with fluorescein isothiocyanate (FITC)-bound goat-antihuman IgG (Sigma, St Louis, MO). The slides were washed once with $0.2 \%$ PBS-Tween, twice with PBS, then fixed with glycerol/PBS (2:1, pH 9.0) and observed under the fluorescent microscope. Light membrane fluorescence and strong, continuously ring-shaped peri-membranous fluorescence were considered positive, while no fluorescence was considered negative.

\section{Collection of Clinical Data}

Routine blood tests included urinalysis, and determination of erythrocyte sedimentation rate, $\mathrm{C}$-reactive protein, and serum levels of complement 3 (C3) and C4. Indirect immunofluorescence was used to determine ANA levels. ELISA and indirect immunofluorescence assays were used to determine the levels of anti-dsDNA antibodies and a positive result on either assay was considered positive for the presence of anti-ds antibodies. Anti-Sm antibody levels were determined by combined double immunodiffusion assay and immunoblotting, and a positive result on either assay was considered positive for the presence of anti-Sm antibodies. AnuA levels were measured by ELISA (all from Tianjin Huayu Biological Technology, Tianjin, China)

\section{Evaluation of Disease Activity Score}

Clinical data of study patients, including presence of skin rash, alopecia, photo allergy, oral ulcers, arthritis, neural injury, and SLEDAI scores were recorded in detail.

\section{Statistical Analysis}

Due to normal distribution, continuous variables were presented as mean and standard deviations, which were compared between the anti-cmDNA negative and positive groups using the Student's $t$-test. The other continuous variables, presented as medians and interquartile ranges (the range between the 25 th and 75 th percentile) due to nonnormal distribution, were compared between the anti-cmDNA negative and positive groups using the Mann-Whitney $U$-test. Categorical variables were expressed as counts and percentages, and compared between different groups by the $\chi^{2}$ test or Fisher's exact test. The McNemar's test was used to measure the consistency of diagnostic sensitivity and specificity among the different antibodies. SAS software package, version 9.2 (SAS Institute, Cary, NC) was used for the statistical analysis. All statistic assessments were evaluated at a two sided $P$ value of 0.05 .

Disclosure: There is no conflict of interest to disclose. 


\section{REFERENCES}

1. Kow NY, Mak A. Costimulatory pathways: physiology and potential therapeutic manipulation in systemic lupus erythematosus. Clin Dev Immunol 2013;2013:245928.

2. Klein-Gitelman M, Reiff A, Silverman ED. Systemic lupus erythematosus in childhood. Rheum Dis Clin North Am 2002;28:561-77, vi-vii.

3. Gualtierotti R, Biggioggero M, Penatti AE, Meroni PL. Updating on the pathogenesis of systemic lupus erythematosus. Autoimmun Rev 2010;10:3-7.

4. Tucker LB, Uribe AG, Fernández M, et al. Adolescent onset of lupus results in more aggressive disease and worse outcomes: results of a nested matched case-control study within LUMINA, a multiethnic US cohort (LUMINA LVII). Lupus 2008;17:314-22.

5. Brunner HI, Gladman DD, Ibañez D, Urowitz MD, Silverman ED. Difference in disease features between childhood-onset and adult-onset systemic lupus erythematosus. Arthritis Rheum 2008;58:556-62.

6. Hersh AO, Trupin L, Yazdany J, et al. Childhood-onset disease as a predictor of mortality in an adult cohort of patients with systemic lupus erythematosus. Arthritis Care Res (Hoboken) 2010;62:1152-9.

7. Chen $\mathrm{YM}$, Lin $\mathrm{CH}$, Chen $\mathrm{HH}$, et al. Onset age affects mortality and renal outcome of female systemic lupus erythematosus patients: a nationwide population-based study in Taiwan. Rheumatology (Oxford) 2014;53: 180-5.

8. Faurschou M, Starklint H, Halberg P, Jacobsen S. Prognostic factors in lupus nephritis: diagnostic and therapeutic delay increases the risk of terminal renal failure. J Rheumatol 2006;33:1563-9.

9. Liang $\mathrm{MH}$, Socher SA, Larson MG, Schur PH. Reliability and validity of six systems for the clinical assessment of disease activity in systemic lupus erythematosus. Arthritis Rheum 1989;32:1107-18.

10. Ching KH, Burbelo PD, Tipton C, et al. Two major autoantibody clusters in systemic lupus erythematosus. PLoS ONE 2012; 7:e32001.

11. Jesus AA, Silva CA, Carneiro-Sampaio M, et al. Anti-C1q antibodies in juvenile-onset systemic lupus erythematosus. Ann N Y Acad Sci 2009;1173:235-8.

12. Binder E, Edelbauer M. Use of biomarkers in the management of children with lupus. Curr Rheumatol Rep 2013;15:312.

13. Servais G, Guillaume MP, Dumarey N, Duchateau J. Evidence of autoantibodies to cell membrane associated DNA (cultured lymphocytes): a new specific marker for rapid identification of systemic lupus erythematosus. Ann Rheum Dis 1998;57:606-13.

14. Su Y, Jia RL, Han L, Li ZG. Role of anti-nucleosome antibody in the diagnosis of systemic lupus erythematosus. Clin Immunol 2007;122:115-20.

15. Ru JL, Zhang LY, Wei H. [Role of anti-nucleosome antibody in the diagnosis of juvenile systemic lupus erythematosus]. Zhonghua Er Ke Za Zhi 2007;45:860-1.

16. Ru JL, Wei H, Lü ZQ, Zhao CY, Li XF. [Role of DNA-associated autoantibodies to cell membrane in the diagnosis of juvenile systemic lupus erythematosus]. Zhonghua Er Ke Za Zhi 2009;47:820-3.
17. Hoffman IE, Lauwerys BR, De Keyser F, et al. Juvenile-onset systemic lupus erythematosus: different clinical and serological pattern than adult-onset systemic lupus erythematosus. Ann Rheum Dis 2009;68:412-5.

18. Baqai T, Ioannou Y. Comparison of juvenile and adult onset systemic lupus erythematosus. Br J Hosp Med (Lond) 2012;73:558-63.

19. Lerner RA, Meinke W, Goldstein DA. Membrane-associated DNA in the cytoplasm of diploid human lymphocytes. Proc Natl Acad Sci USA 1971;68:1212-6.

20. Chen HY, Guo JL, Li ZG. Significance of anti-cell membrane-associated DNA (mDNA) antibodies in systemic lupus erythematosus. Clin Rheumatol 2008;27:183-7.

21. Bennett RM, Davis J, Campbell S, Portnoff S. Lactoferrin binds to cell membrane DNA. Association of surface DNA with an enriched population of B cells and monocytes. J Clin Invest 1983;71:611-8.

22. Meinke W, Hall MR, Goldstein DA, Kohne DE, Lerner RA. Physical properties of cytoplasmic membrane-associated DNA. J Mol Biol 1973;78: 43-56.

23. Wu JF, Yang YH, Wang LC, Lee JH, Shen EY, Chiang BL. Antinucleosome antibodies correlate with the disease severity in children with systemic lupus erythematosus. J Autoimmun 2006;27:119-24.

24. Watson L, Beresford MW. Urine biomarkers in juvenile-onset SLE nephritis. Pediatr Nephrol 2013;28:363-74.

25. Morgan TA, Watson L, McCann LJ, Beresford MW. Children and adolescents with SLE: not just little adults. Lupus 2013;22:1309-19.

26. Malleson PN, Sailer M, Mackinnon MJ. Usefulness of antinuclear antibody testing to screen for rheumatic diseases. Arch Dis Child 1997;77: 299-304.

27. Bennett RM, Cornell KA, Merritt MJ, Bakke AC, Hsu PH, Hefeneider SH. Autoimmunity to a $28-30 \mathrm{kD}$ cell membrane DNA binding protein: occurrence in selected sera from patients with SLE and mixed connective tissue disease (MCTD). Clin Exp Immunol 1991;86:374-9.

28. Hefeneider SH, Bennett RM, Pham TQ, Cornell K, McCoy SL, Heinrich MC. Identification of a cell-surface DNA receptor and its association with systemic lupus erythematosus. J Invest Dermatol 1990;94(6 Suppl):79S-84S.

29. Chen HY, Jia RL, Li ZG, et al.Determination of anti-DNA antibodies and the cell membrane in the diagnosis of systemic lupus erythematosus. J Rheumatol 2005;229-33.

30. Shi C, Jia RL, Han L, et al. Anti-membrane DNA autoantibodies rapid detection of systemic lupus erythematosus. Chin J Rheumatol 2005; 227-9.

31. Hua W, Lu ZQ, Ru JL, Li XF, Xu K, Wen HY. [The detection of DNAassociated autoantibodies to cell membrane and its combination with other autoantibodies in the diagnosis of systemic lupus erythematosus]. Chin J Rheumatol 2010;14:185-7.

32. Guo JL, Bi LQ, Li ZG. [Significance of anti-cell membrane associated DNA antibodies in diagnosis of systemic lupus erythematosus]. Chin J Lab Med 2006;9:787-90. 